\title{
Kernos
}

Revue internationale et pluridisciplinaire de religion grecque antique

16 | 2003

Varia

\section{R. DI DONATO, Hierà. Prolegomena ad uno studio storico anthropologico della religione greca}

\section{Vinciane Pirenne-Delforge}

\section{(2) OpenEdition \\ Journals}

\section{Édition électronique}

URL : http://journals.openedition.org/kernos/859

DOI : $10.4000 /$ kernos.859

ISSN : 2034-7871

\section{Éditeur}

Centre international d'étude de la religion grecque antique

\section{Édition imprimée}

Date de publication : 1 janvier 2003

Pagination : 389-390

ISSN : 0776-3824

\section{Référence électronique}

Vinciane Pirenne-Delforge, « R. Di Donato, Hierà. Prolegomena ad uno studio storico anthropologico della religione greca », Kernos [En ligne], 16 | 2003, mis en ligne le 14 avril 2011, consulté le 22 septembre 2020. URL : http://journals.openedition.org/kernos/859; DOI : https://doi.org/10.4000/ kernos.859 
J. Geffcken, Die Oracula Sibyllina (Die griechischen christlichen Scbriftsteller der ersten drei Jabrbunderte), Leipzig, 1902, p. 153-157; on notera que ce fragment ne se lit ni dans l'édition de H. Erbse, ni dans celle de P.F. Beatrice, bien qu'une grande section y soit consacrée aux oracles des Sibylles; toutefois, H. Erbse, Fragmente, p. 97 n. 188, et p. 188, dit que ce fragment, que nous avons également découvert dans l'Atheniensis, Metochion Panagiou Tapbou $363, f .136^{\mathrm{V}}$, accompagne souvent ce qui suit dans notte manuscrit. En

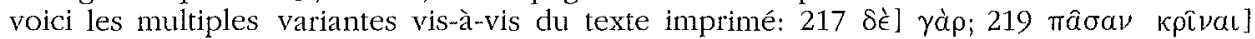

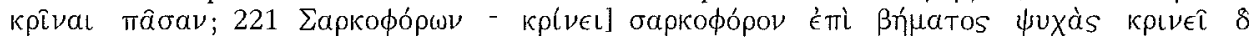

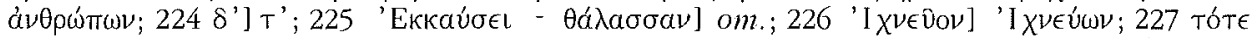

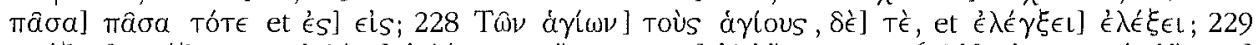

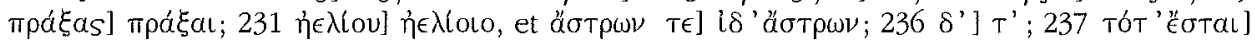

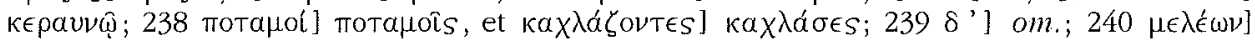

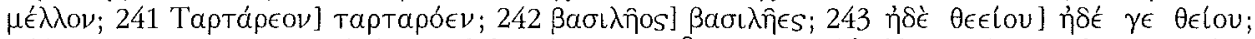

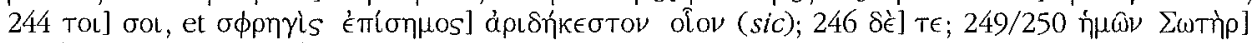

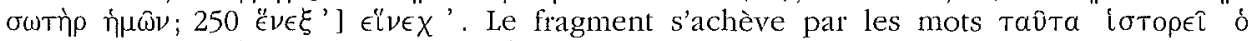

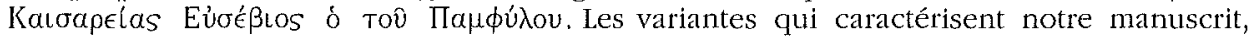
montrent que l'Athous se rapproche de la version de l'oracle telle qu'elle est conservée dans l'Oratio Constantini imperatoris ad sanctorum coetum d'Eusèbe de Césarée (Clavis Patrum Graecorum 3497); voir l'édition d'I.A. Heikel, Eusebius Werke, I, Über das Leben Constantins. Constantins Rede an die beilige Versammlung. Tricennatsrede an Constantin. (Die griechischen christlichen Schriftsteller der ersten drei Jabrbunderte), Leipzig, 1902, \$ XVIII (p. 179-181).

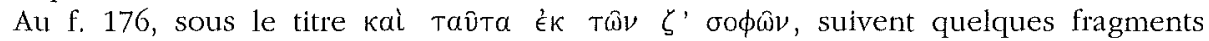
appartenant à la collection $\pi$ de la $\Theta \epsilon o \gamma \nu \omega o t a$; en voici l'inventaire détaillé: $\pi 7$ (2 vari-

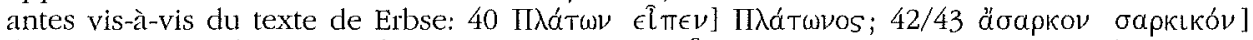

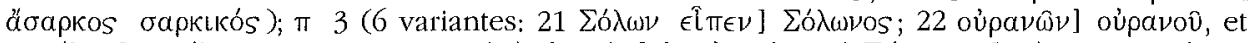

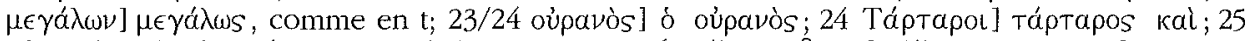

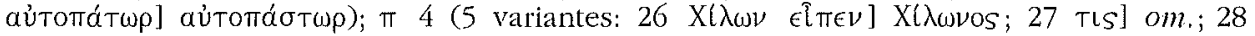

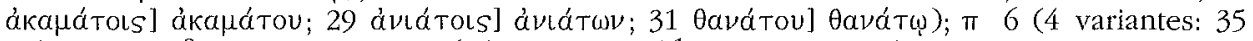

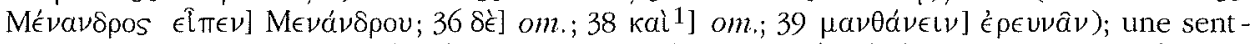

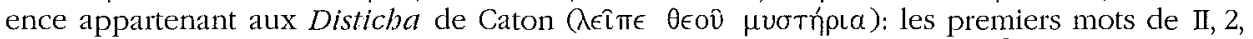
dans la traduction de Maxime Planude $\left({ }^{1}\right) ; \pi 2$ (2 variantes: 14 Blas $\epsilon[\pi \epsilon \nu]$ Bíavtos; 16

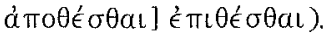

Pour la collection $\pi, l^{\prime}$ Atbous est clairement apparenté à la famille $t$ (voir Erbse, Fragmenta, p. xxx, stemma).

Toujours au f. 176, on lit encore quelques sections faisant partie de la collection w:

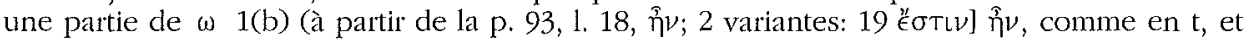

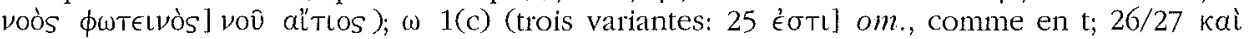

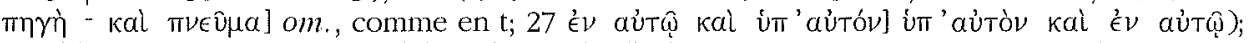
$\omega$ 1(e) (aucune variante); le début de $\omega$ 1(f) (le bas de la page étant découpé, le texte de ce dernier fragment est presque illisible; le dernier mot qu'on puisse y lire est $\pi \rho \hat{T} T \mathrm{v}$, au

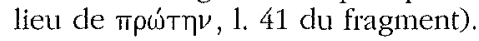

Pour la collection $\omega$, l'Athous est clairement apparenté à la famille t (pour la désignation $t$ utilisée pour la recension w, voir Erbse, Fragmenta, p. 92).

\section{(Katholieke Universiteit Leuven)}

Ricardo Di Donato, Hierà. Prolegomena ad uno studio storico antbropologico della religione greca, Università di Pisa, Edizioni Plus, 2001. 1 vol. $17 \times 24 \mathrm{~cm}, 368 \mathrm{p}$. (Didattica e ricerca. Manuali). ISBN : 88-8492-091-4.

Voici un manuel résolument peu conventionnel. Plutôt que d'offrir le panorama habituel de «tutte le verità di cui era necessaria la conoscenza » (p. 13), l'A. se place dans une perspective essentiellement historiographique et problématique. Nourri de l'approche « anthropologique » française de M. Mauss, L. Gernet et J.-P. Vernant, cet ouvrage invite

1 Voir l'édition de V. Ontoleva, Maximus Planudes. Disticha Catonis in Graecum translata, Roma, 1992 (Bibliotbeca Atena, 28), p. 8. 
son utilisateur à un parcours de lecture, truffé d'extraits et de résumés de travaux sur la religion grecque, et de commentaires plus ou moins critiques selon les cas. Une première partie intitulée "Studiare la religione grecal " s'ouvre avec quelques brèves considérations linguistiques sur la notion grecque de «sacré » (mais sans discuter Rudhardt, ce qui est dommage), passe de L. Gernet et J.-P. Vernant à W. Burkert, W.F. Otto, K. Kerényi et A. Brelich. L'orientation générale de cette première partie relève de l'opposition entre un point de vue fondé sur l'histoire - sur une anthropologie historique - et celui qui postule l'invariance de l'esprit humain dont il recherche les permanences. La deuxième partie («L'espressione religiosa dei Greci delle origini ») se tourne vers la recherche des commencements qui caractérise bon nombre de travaux sur la religion grecque. Balayant d'un revers de plume les postulats du Trattato di Antropologia del sacro dirigé par J. Ries, notamment la contribution de $R$. Boyer sur les indo-européens, il fait le point sur les données religieuses «mycéniennes » avec l'ouvrage déjà ancien de M. Gérard-Rousseau, et une présentation des données par $\mathrm{A}$. Brelich, brève mais remarquable, et les derniers développements avec P. Darcque. Pour cette mise au point, il est clair qu'il faudra désormais tenir compte des dernières découvertes de Thèbes, publiées par V.L. Aravantinos, L. Godart et A. Sacconi ${ }^{1}$. La suite attendue de ces développements sur les « origines »se trouve évidemment dans les travaux de M.P. Nilsson. Tout juste après, un curieux développement sur la prière dans l'Iliade rompt avec l'orientation historiographique adoptée jusque-là. Repartir de la thèse de D. Aubriot sur le sujet (Lyon, 1992) aurait peutêtre permis de conserver la structure expositive de l'ensemble tout en soutenant la volonté de l'A. de se fonder sur un texte ancien déterminé. Le fil historiographique reprend ensuite, avec G. Murray, longuement analysé, A. Brelich et les orientations plus philosophiques de G. Cambiano. La troisième et dernière partie s'intitule « La religione degli Ateniesi. L'interpretazione più recente » et livre en fait une très longue présentation critique (près de 100 pages) de l'ouvrage de R. Parker, Atbenian Religion. A History (Oxford, 1996), avec un petit appendice sur le procès de Socrate analysé par G. De Sanctis. Une bibliographie très fournie referme ce manuel, ainsi qu'un index des noms antiques et des auteurs (modernes) cités. La fin un peu abrupte de l'ouvrage est à l'image de la fin souvent tout aussi abrupte des présentations d'auteurs. Mais sans doute est-ce délibéré : ce parcours est avant tout une mine de réflexions qui donne à penser sur nos propres manières de travailler dans le domaine.

Vinciane Pirenne-Delforge (FNRS - Université de Liège)

Giovanni Casadio (éd.), Ugo Bianchi. Una vita per la Storia delle Religioni, Roma, "Il Calamo", 2002. 1 vol. $17 \times 24$ cm, 524 p. (Biblioteca di Storia delle Religioni, 3). ISBN : 88-88039-24-4.

Sont à l'origine de cet imposant volume deux journées d'étude qui, sous le même intitulé, ont été organisées à l'Université de Salerne, les 14 et 15 avril 1997, à l'initiative de deux disciples, $O$. Bucci et $G$. Casadio. La première de ces journées coïncidait, jour pour jour, avec le deuxième anniversaire du décès d'Ugo Bianchi, né en 1922 et mort inopinément à Rome. Cette manifestation d'hommage était placée sous le patronage de la Société italienne d'histoire des religions, de l'Association internationale pour l'histoire des religions (I.A.H.R.), dont U. Bianchi assurait la présidence au moment de son décès, et du Département des sciences de l'Antiquité de l'Université de Saleme. La vingtaine de contributions qui composent l'ouvrage sont réparties dans trois parties principales. La première, précédée d'une introduction de l'éditeur, est consacrée à la biographie du défunt et évoque plus particulièrement ses activités d'enseignement et de recherche aux Universités de Messine, Bologne et Rome; P. Antes, actuel président de l'I.A.H.R., met en relief sa longue collaboration à cette Association et le rôle important qu'il y joua. L'œuvre d'Ugo Bianchi fait l'objet de la deuxième partie; on y dresse le bilan de ses études portant sur les religions de la Mésopotamie, de l'Iran, de l'Égypte, de la Grèce et de Rome, de ses recherches aussi relatives aussi au christianisme, au gnosticisme, au manichéisme, au

1 Thèbes. Fouilles de la Cadmée I. Les tablettes en Linéaire B de la Odos Pelopidou. Édition et commenlaire, Pisa/Roma, IPE, 2001, spéc. p. 317-325. 